\title{
Development of an Automatic System for Feasibility Studies on Transport Investment
}

\author{
Jin Ki Eom ${ }^{\dagger}$, Kwang Sub Lee*, Jun Lee* and Dae Seop Moon**
}

\begin{abstract}
New transport technologies have been rapidly developed and various transport systems are ready to introduce. However, there no exists effective decision making tools for pre-feasibility studies on transport systems. The pre-feasibility studies should provide reliable solutions about whether a certain transport system is feasible or not by cost-benefit analysis and effectiveness test of enhancing regional economy. This study introduces the smart transport investment/information system (SMARTIS) for supporting decision making with ease, fast and reliable methods. The SMARTIS incorporates travel demand estimates into the process of feasibility studies with automatic data processing methods to give reliable solutions quickly. The SMARTIS is expected to be informative for transit agencies, planners, and operators.
\end{abstract}

Keywords : Feasibility study, Travel demand, Benefit/Cost, User Interface

\section{Introduction}

As new technologies are developed, the business of transportation systems rapidly increases with large amount of monetary fund on R\&D scheme in Korea. The new transport systems currently ready to implement are bimodal tram, wireless tram, PRT (personal-rapid transit), TTX(tilting train), etc.

The engineers involved in design and development of new transport systems need to seriously consider what target passengers to transport using new systems and where the systems are beneficial within public transport systems at an initial development step. Without considering how to implement new transport systems before developing them, the system developers are hard to find right place to implement after completion of system.

Further current decision-making process on transport investment is based on government oriented guideline which mainly provides the methods of travel demand analysis and benefit-cost analysis with various indexes. This

\footnotetext{
$\dagger$ Corresponding author: Transport Systems Research Team, KRRI, Uiwang 437757, Korea

E-mail : jkom00@krri.re.kr

* Transport Systems Research Team, KRRI, Uiwang 437-757, Korea

${ }^{* *}$ Convergence Technology Research Team, KRRI, Uiwang 437-757, Korea
}

guideline is appropriate to provide feasibility of whole transport system investment at long-term periods such as thirty or forty years including construction and operation periods. Therefore, the guideline seems to be heavy for transport planners to practically use in pre-feasibility studies due to its complex procedure in each step, and provides general cost units of transport systems such as vehicle cost, construction cost, operation cost, passenger capacity, which do not well represent the specifications of new transport systems.

In order to address these issues, this study try to develop a decision-making system by comprising several light decision processes which are expected to be helpful for transport planners by providing quick and relatively precise solution. In this study, we call the system as SMARTIS (Smart Transport Investment/Information System) and introduce the direction of system development and provide general concept of the system.

\section{SMARTIS (Smart Transport Investment System)}

Unlike existing feasibility study the peculiarity of SMARTIS is a kind of pre-feasibility decision-making support system on supposition of implementation of various transport systems including new transport systems. 


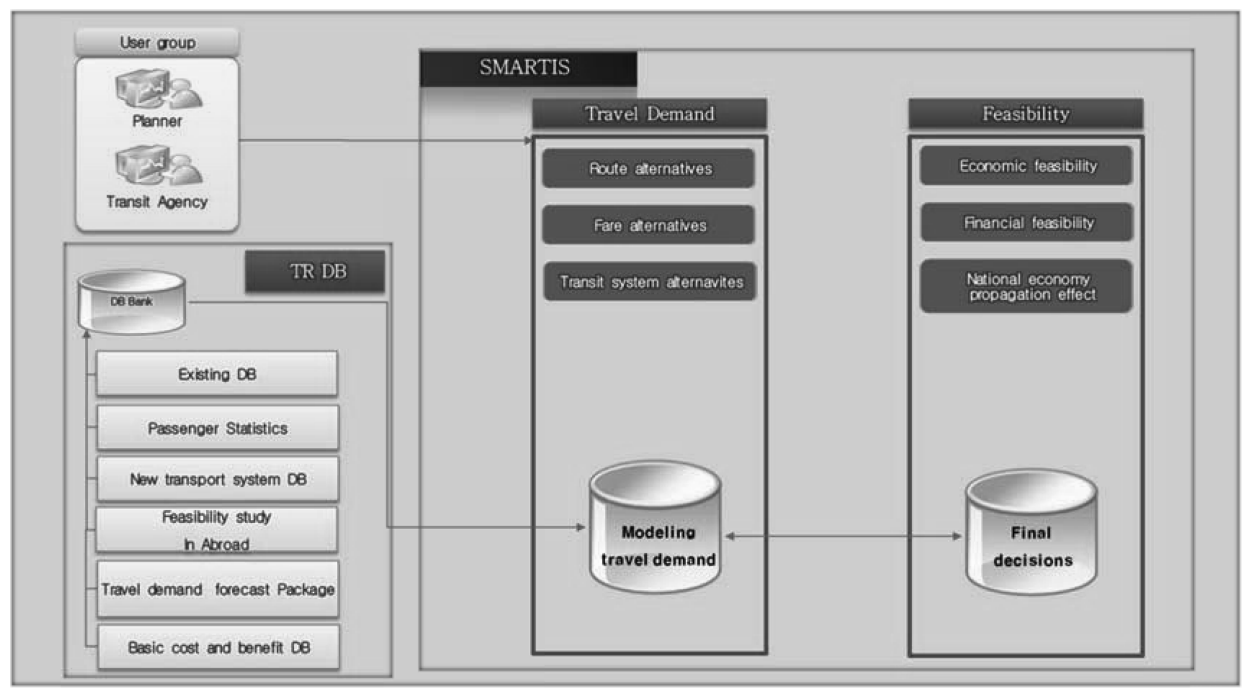

Fig. 1 Data and system structure of SMARTIS

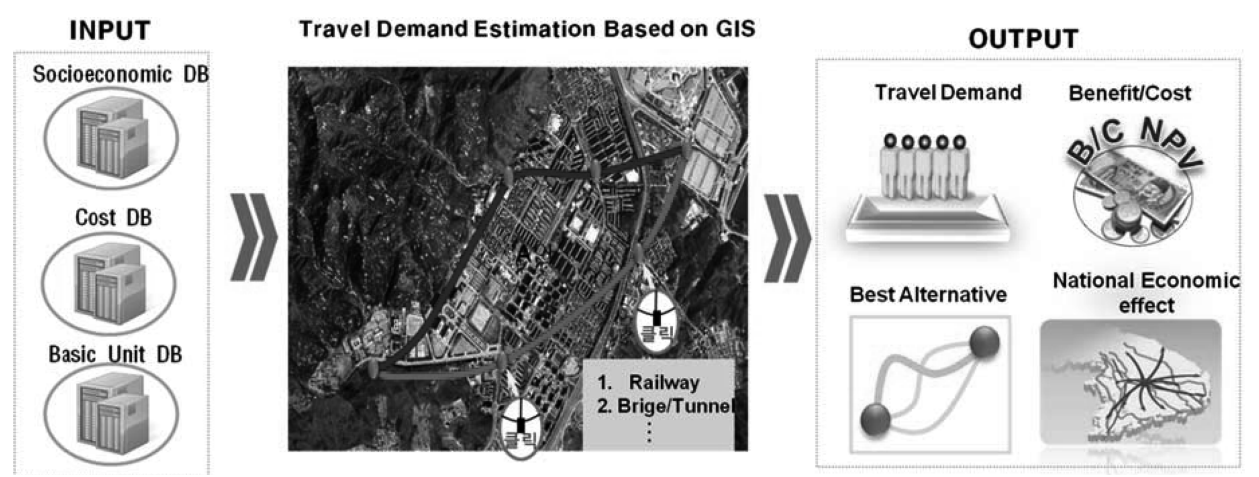

Fig. 2 Direct demand estimation of SMARTIS

The goal of this system is to develop quick and precise decision system to help planners on the decisions of which system is the best fit to a certain region and its economic feasibilities, and various conditions of new transport system development. When the system is successfully developed, the system will be useful to make better decisions on transport systems.

\subsection{System outline}

For the simple and quick response to investment scenarios, the SMARTIS consists of three parts: 1) transportation database, 2) travel demand analysis, and 3) feasibility analysis as shown in Fig. 1.

\subsubsection{Basic database}

The database includes observed passenger volumes, socio-economic data such as population and employees by transportation analysis zone (TAZ) level, and output of previous feasibility studies, accomplished recent ten years in Korea, and characteristics of new transport system regarding the passenger capacity, operational speed, and cost of vehicle, construction, and operation. Specially, the previous reports of feasibility studies on recent highway and railway projects give beneficial information about the relationship between regional characteristics and feasibility studies like local population constitution, travel demand and local land use patterns and feasibility of transportation system. The SMARTIS will be able to adopt this useful relationship to modify the result of pre-feasibility study result.

\subsubsection{Demand analysis}

The travel demand analysis approaches needs to be two parts: one is a traditional four-step approach which is based on national transport database providing origin-destination (OD), and another approach is simple method to provide general travel demand estimation. When the travel demand is estimated with given travel OD table, the well- 
known commercial programs such as TransCAD and EMME $/ 3$ can be used to produce travel demand estimation which will be the input of SMARTIS to calculate benefit and cost for a decision of economic feasibilities. In case of OD table not given from the database, the demand can be estimated based on the form of direct demand modeling structure. As shown in Fig. 2. The concept of direct demand estimation, for example, is to set up a transit route by defining stations and routes on the GIS-based map, and then the direct demand model provides the number of passengers in each station based on socio-economic database. Once this can be accomplished this system saves huge amount of time for forecasting travel demand compared to traditional demand analysis.

In order to enhance the ability of precise prediction, the data of traffic volume and ridership will be useful to validate and modify the estimates of travel demand. Further the network calibration is crucial in reliable travel demand forecasting. Therefore, the methods of calibration and validation are internally stored in the system.

\subsubsection{Feasibility measures}

With respect to feasibility studies, the measures for economic feasibility are defined as benefit-cost $(\mathrm{B} / \mathrm{C})$ ratio, and net present value (NPV), and internal rate of return (IRR). These values are calculated by the function of travel demand, which is well reported at the fifty edition of national guidebook of feasibility studies for highway and railway project developed by Korea Development Institute (KDI). One limitation of the guidebook is that the railway system is considered only regular system so that the various new systems developed currently do not have system related cost and benefit. Hence, we will provide the cost values related to the new system and explore new benefits as well.

\section{SMARTIS (Smart Transport Information System) UI}

In order to properly analyze feasibility studies, the user interface (UI) is greatly important. The user interface has to be set up by progress step of feasibility studies. The UI will have the five steps of user interface: 1) input the basic information about a project such as vehicle system and target area for the study, 2) input the definition of study year and scenarios of the project with respect to route plan, vehicle system, and operation alternatives, 3) decision of methodology for travel demand estimation as either conventional method or direct demand method. If user chooses the conventional method, the travel demand will be estimated by using commercial software outer of

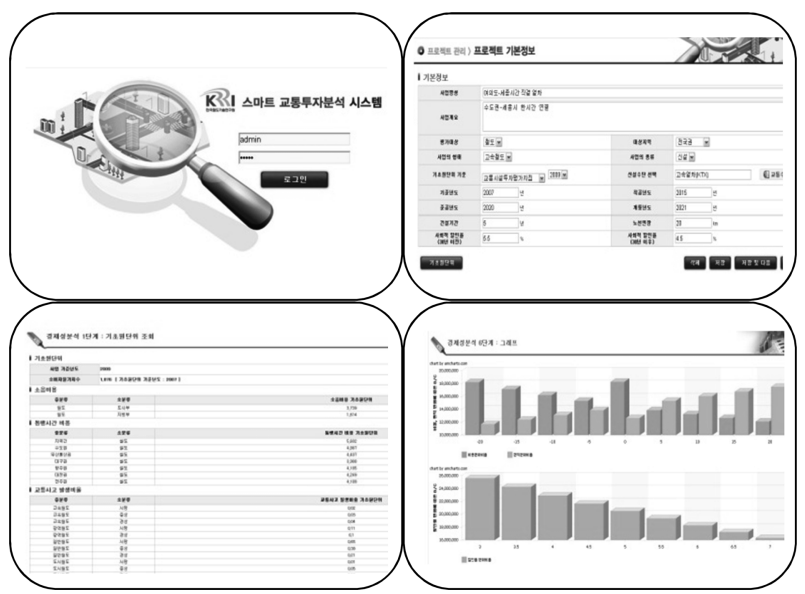

Fig. 3 SMARTIS UI (Prototype)

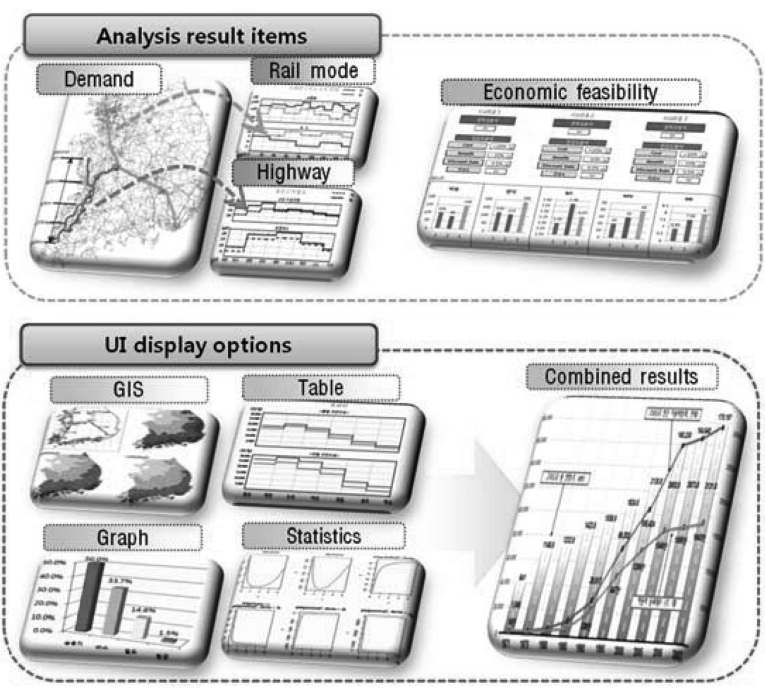

Fig. 4 Output of SMARTIS

SMARTIS then the estimated travel demand will be input in the system, 4) calculation of benefit and cost for the analysis project. The selection of benefit and cost items will call the unit values from the database, 5) feasibility examination by measures of effect such as benefit-cost ratio, internal rate of return, net-present value, etc is accomplished. If the economic measures do not affect on feasibility studies then the national economy propagation effect analysis will be complete. When these steps are connected with each other, the data for input and output are complex to understand the system. Therefore, the system has to be designed for users to understand easy and simple to see the result. To do this, the manual has to be ready to users. Fig. 3 shows the prototype of user interface of SMARTIS. The UI will be operated under the web-based environment accessed by user ID and password. The goal 
Jin Ki Eom, Kwang Sub Lee, Jun Lee and Dae Seop Moon / IJR, 5(4), 144-147, 2012

of UI system is to allow users who may not have special knowledge about feasibility study to easily access and get the results of their transport project alternatives.

Fig. 4 shows the expected results provided by SMARTIS. The basic results will be travel demand, economic feasibility, and national economy propagation effect by project alternatives as user initially defined. The UI will give clear and simple results by using display options such as GIS, table, graph, statistics of study region and route, and combined results according to user definition.

\section{Conclusions}

This study introduces the direction of development of the smart transport information system (SMARTIS) for supporting decision making with ease, fast and reliable methods. The SMARTIS will be a useful tool for pre-feasibility studies with respect to new technologies on transport systems. The SMARTIS incorporates travel demand estimates into the process of feasibility studies with automatic data processing methods to give reliable solutions quickly. SMARTIS consists of transportation database, travel demand analysis, and feasibility analysis. The user interface (UI) is set up by progress step of feasibility studies consisting of five steps as input the basic information about a project, input the definition of study year and scenarios of the project with respect to route plan, vehicle system, and operation alternatives, decision of methodology for travel demand estimation as either conventional method or direct demand method, calculation of benefit and cost for the analysis project, and feasibility examination by measures of effect such as benefit-cost ratio, internal rate of return, net-present value, etc. The SMARTIS is expected to be informative for transit agencies, planners, and operators.

\section{References}

1. Usvy (2009). "Sketch Model to Forecast Heavy-Rail Ridership," Proceedings of 88th Transportation Research Board conference.

2. CalTrans (2002). http://www.dot.ca.gov/hq/tpp/offces/ote/LCBC Analysis_Model.html

3. KTDB. (2012). Korea Transport DataBase. Available at http://ww.ktdb.go.kr

4. TCRP (2002). Estimating the Benefits and Costs of Public Transit Projects: A Guidebook for Practitioners.

5. Greenbook (2012). http://www.hm-treasury.gov.uk/data greenbook_index.htm.

Received(November 12, 2012), Accepted(November 24, 2012) 